\title{
Artificial neural network approach for acute poisoning mortality prediction in emergency departments
}

\author{
Seon Yeong Park', Kisung Kim² , Seon Hee Woo ${ }^{3}$, Jung Taek Park ${ }^{4}$, \\ Sikyoung Jeong ${ }^{1}$, Jinwoo Kim ${ }^{5}$, Sungyoup Hong ${ }^{1}$ \\ 'Department of Emergency Medicine, Daejeon St. Mary's Hospital, The Catholic University of Korea College \\ of Medicine, Daejeon, Korea \\ ${ }^{2}$ BioBrain Inc., Daejeon, Korea \\ ${ }^{3}$ Department of Emergency Medicine, Incheon St. Mary's Hospital, The Catholic University of Korea College \\ of Medicine, Incheon, Korea \\ ${ }^{4}$ Department of Emergency Medicine, Uijeongbu St. Mary's Hospital, The Catholic University of Korea \\ College of Medicine, Uijeongbu, Korea \\ ${ }^{5}$ Department of Emergency Medical Service, Daejeon Health Institute of Technology, Daejeon, Korea
}

Objective The number of deaths due to acute poisoning (AP) is on the increase. It is crucial to predict AP patient mortality to identify those requiring intensive care for providing appropriate patient care as well as preserving medical resources. The aim of this study is to predict the risk of in-hospital mortality associated with AP using an artificial neural network (ANN) model.

Methods In this multicenter retrospective study, ANN and logistic regression models were constructed using the clinical and laboratory data of 1,304 patients seeking emergency treatment for AP. The ANN model was first trained on 912/1,304 (70\%) randomly selected patients and then tested on the remaining 392/1,304 (30\%). Receiver operating characteristic curve analysis was used to evaluate the mortality prediction of the two models.

Results Age, endotracheal intubation status, and intensive care unit admission were significant predictors of mortality in patients with AP in the multivariate logistic regression model. The ANN model indicated age, Glasgow Coma Scale, intensive care unit admission, and endotracheal intubation status were critical factors among the 12 independent variables related to in-hospital mortality. The area under the receiver operating characteristic curve for mortality prediction was significantly higher in the ANN model compared to the logistic regression model.

Conclusion This study establishes that the ANN model could be a valuable tool for predicting the risk of death following AP. Thus, it may facilitate effective patient triage and improve the outcomes.

Keywords Poisoning; Mortality; Neural networks, computer; Logistic models; Prognosis

Received: 26 August 2020

Revised: 20 October 2020

Accepted: 29 October 2020

Correspondence to: Sungyoup Hong Department of Emergency Medicine, Daejeon St. Mary's Hospital, The Catholic University of Korea College of Medicine, 64 Daeheung-ro, Jung-gu, Daejeon 34943, Korea

E-mail: emhong@catholic.ac.kr ORCID

https://orcid.org/0000-0002-3387-6194

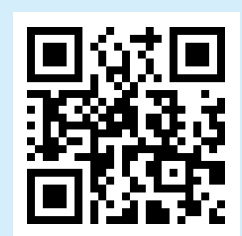

How to cite this article: Park SY, Kim K, Woo SH, Park JT, Jeong S, Kim J, Hong S. Artificial neural network approach for acute poisoning mortality prediction in emergency departments. Clin Exp Emerg Med 2021;8(3):229-236. https:// doi.org/10.15441/ceem.20.113
This is an Open Access article distributed under the terms of the Creative Commons Attribution Non-Commercial License (https:// creativecommons.org/licenses/by-nc/4.0/). 


Capsule
What is already known
The number of deaths from acute poisoning accounts for a significant proportion of deaths from external causes. The
poisoning severity score is a good predictor of mortality. However, the poisoning severity score needs to consider the
overall clinical progression, and the clinician needs to collect a large amount of data from the 12 organ systems. There-
fore, a new scoring tool that is a simple and efficient prognosis prediction system for high throughput clinical data is
required.
What is new in the current study
A novel artificial neural network model was developed to measure the risk of death in patients presenting with acute
poisoning on initial emergency department assessment. Trained artificial neural networks approach the functionality of
a cluster in a fundamental manner, which can inform triage in emergency departments more effectively than logistic
regression to provide an additional tool in efficient resource and human resource management.

\section{INTRODUCTION}

The number of deaths due to acute poisoning (AP) because of drug or substance ingestion tripled from 6,100 in 1999 to 36,500 in 2008 in the United States of America, exceeding the deaths due to traffic accidents. ${ }^{1}$ Globally, unintentional AP caused 86,400 deaths at a rate of 1.2 per 100,000 individuals in 2015. Patients suspected of AP account for a substantial proportion of the visitors to emergency departments (EDs). ${ }^{2}$

In general, patients with AP have a mortality of $1 \%$ to $3 \%$, which is lower compared to those of other diseases needing intensive care. ${ }^{3}$ Clinical staff prefer the hospitalization of patients with AP in the intensive care unit (ICU) in case of additional risks, such as potential suicide attempts. ${ }^{4}$ Nevertheless, it is crucial to predict patient mortality in order to identify those requiring intensive care for providing appropriate patient care as well as preserving medical resources. The development of tools that can stratify the severity of AP, based on clinical and laboratory parameters, can improve the approach and treatment of this condition, particularly in EDs with limited facilities.

A logistic regression (LR) model is the most typical means of predicting the risk of binary outcomes occurring due to risk factor exposure; however, this model has poor predictability for data based on small sample sizes. ${ }^{5}$ Although King and Zeng ${ }^{6}$ offer an extensive discourse on rare event data, substantial misconceptions remain on this issue.

In comparison, artificial neural networks (ANNs), which attempt to emulate neuronal networks in the human brain, have been created to address classification and prediction problems, and have particular relevance and application in medical science. ${ }^{7}$ Although the ANN has been utilized as an alternative approach to $L R$, similarities in the statistical procedures exist between the two models. However, the predictor variables in a neural network (NN) usually undergo nonlinear transformation through each hidden and output node. Thus, an NN can generate a model with nonlinear relationships having substantially greater complexity than those in an LR model. Furthermore, ANNs can detect any complex nonlinear relationship between independent and dependent variables. ${ }^{8}$

Data to support the replacement of standard statistical approaches by ANNs as the method of choice for the classification of medical data are insufficient. ${ }^{9}$ Hence, both methods should continue to be used and explored in a complementary manner. To the best of our knowledge, there are no studies on the application of ANNs for the prediction of rare and fatal events in critical diseases, such as AP. In this study, the performances of general regression and ANN analysis are compared using data collected over several years from patients presenting to EDs with AP. Trained ANNs approach the functionality of a cluster in a fundamental manner, which we expect can perform triage in EDs as effectively as $L R$, and provide an additional tool for efficient resource and human resource management. ${ }^{7}$

\section{METHODS}

\section{Design and data collection}

This study was designed as a multicenter, retrospective, and medical record review of patients with AP. Patients older than 15 years, who visited the emergency center of two tertiary care university hospitals in South Korea (Incheon St. Mary's Hospital and Yeouido St. Mary's Hospital) within 24 hours of drug or toxic substance ingestion, between January 2010 and December 2016, were enrolled in the study. Patients with poisoning associated with a suicide attempt or accidental exposure were also included. The study 
protocol was approved by the institutional review board of the Catholic Medical Center of Korea (DC20ZIS10034) and written informed consent was waived as all the data were retrospectively collected through retrospective chart review. The initial AP severity was classified based on the worst poisoning severity score (PSS) during the first 24-hour period in the ED. ${ }^{10}$ The initial PSS was measured immediately on arrival at the ED by an ED clinician, whereas the final PSS was determined as the highest score during the entire hospital stay. For patients who were discharged or had died during the first 24-hour period, the PSS was checked once.

Patient monitoring and laboratory measurements were performed as part of routine patient management in the ED, and immediate appropriate medical care was offered for the clinical condition by the emergency medical personnel.

\section{Classification of poisoning substances and risk point estimation in multiple toxins ingestion}

To estimate the toxic effects in patients who had ingested multiple toxins simultaneously, we estimated the sum of risks of the poisonous substances. These substances were classified according to the ICD-10 (International Statistical Classification of Diseases and Related Health Problems) (T36-T50). ${ }^{11}$ LR was performed by incorporating the number of ingested poisons in each class of toxic material as an independent variable and in each PSS group (high [3 or 4] or low [0-2] risk) as a dependent variable. The maximum likelihood estimation value of each class of poison obtained through $L R$ was considered a risk point, and the sum of the risk points (SRP) for all the ingested toxic substances was considered as the predicted risk of death for patients with multiple poisons ingestion. The calculated SRP was employed as one of the independent variables in univariate and multivariate $L R$, and ANN analysis was used to predict the mortality risk factors following mixed substance intake.

\section{LR analysis}

To identify the potential predictors of in-hospital death following $A P$, univariate $L R$ analyses were performed incorporating the demographic informations, clinical characteristics, and laboratory variables. Multivariate LR analysis was performed for a combination of selected risk factors (screened in univariate analyses) exhibiting P-values less than 0.20 . Results of the regression analyses were presented as odds ratios (ORs) with the accompanying 95\% confidence intervals ( $\mathrm{Cls}$ ).

\section{Backpropagation ANN modeling}

All the data were randomly divided into training and test datasets

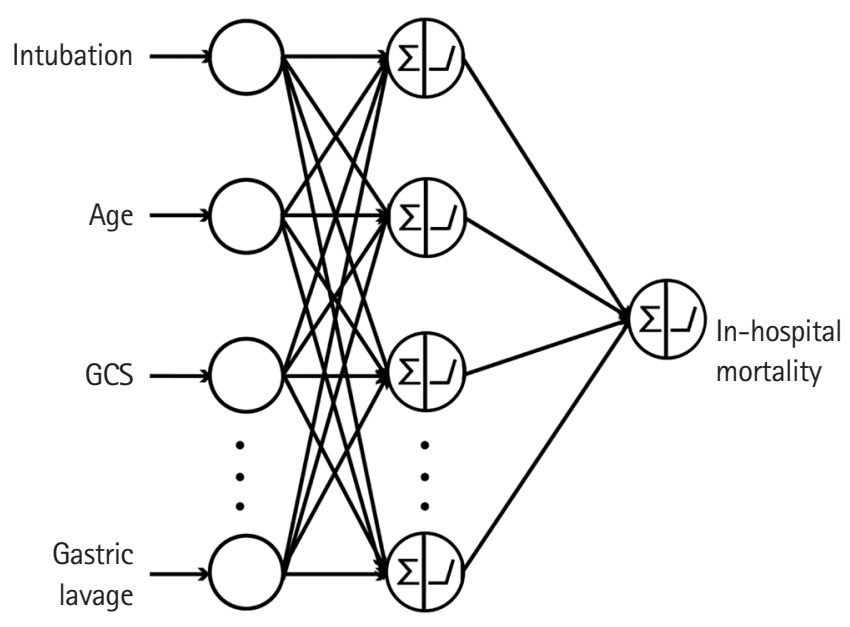

Fig. 1. Schematic diagram of the backpropagation artificial neural network architecture used to predict the probability of in-hospital mortality. The input layer contained 12 neurons and the hidden layers were 30 with 12 neurons. The output layer has one node (in-hospital mortality). GCS, Glasgow Coma Scale.

at a ratio of 7:3. The backpropagation (BP) ANN model comprised three layers, input, hidden, and output, containing 14, 30, and 1 neuron, respectively. A total of 12 input variables, including age of the patient, diastolic blood pressure, pulse rate, Glasgow Coma Scale (GCS), PSS, PSS group, admission to the ICU, length of ICU stay, endotracheal intubation status, diabetes mellitus, and gastric lavage were employed in the model. All the hidden layers were fully connected, and the rectified linear unit was used as the activation function for each neuron in this layer (Fig. 1)..$^{12}$ The single output layer neuron indicated 1 or 0 for death or survival, respectively. The Relieff algorithm was used for feature ranking and selecting the relevant features (ReliefF $>0.2$ ).

\section{Statistical procedures}

Continuous data were presented as the median and interquartile range (IQR), whereas dichotomous data were presented as the number and percentage. The significance of differences between the two groups were evaluated through the unpaired Wilcoxon rank-sum test for continuous variables, whereas the chi-squared test or Fisher exact test, as appropriate, was applied for dichotomous variables. LR analysis and the ANN model were developed for predicting the occurrence of mortality in patients with AP. The ANN structure included 30 hidden layers, and each hidden layer had 12 nodes. The receiver operating characteristic (ROC) curves were plotted, and the empirical method of Patel and Goyal. ${ }^{7}$ was used to compute the area under the curve. The Wilcoxon signedrank test statistic, based on pairwise comparisons, was used to determine the differences between the area under the curves of the two statistical methods. P-values $<0.05$ were considered 
statistically significant. The statistical analyses were performed using Orange ver. 3.23, an open-source machine learning toolkit (University of Ljubljana, Ljubljana, Slovenia), and RStudio ver. 1.2.5033 (RStudio Inc., Champaign, IL, USA).

\section{RESULTS}

\section{Patient characteristics}

A total of 1,304 poisoned patients ( $>15$ years) had visited the ED during the previously mentioned study period. Among these, 434 men and 870 women were included in the study. Table 1 lists the general characteristics of the study population group. A total of 12 patients died in the ED or following hospital admission. The median age of ED arrival was 44 years (range, 15-96 years; IQR, 29-57) in the survival group, which was significantly lower than that of the death group at 72 years (IQR, 57-75). Patients in this "death" group had visited the ED after ingesting $1.8\left(I_{1}, 1-2\right)$

Table 1. Descriptive statistics by mortality after acute poisoning

\begin{tabular}{|c|c|c|c|}
\hline Variable & $\begin{array}{c}\text { Survival } \\
(n=1,292)\end{array}$ & $\begin{array}{l}\text { Death } \\
(n=12)\end{array}$ & P-value \\
\hline Age & $44(29-57)$ & $72(57-75)$ & $<0.001$ \\
\hline Sex & & & 0.352 \\
\hline Female & $864(66.9)$ & $6(50.0)$ & \\
\hline Male & $428(33.1)$ & $6(50.0)$ & \\
\hline No. of poisoning substances & $2.2(1-3)$ & $1.8(1-2)$ & 0.410 \\
\hline \multicolumn{4}{|l|}{ Vital signs } \\
\hline Systolic blood pressure & 122 (108-139) & $127(113-138)$ & 0.810 \\
\hline Diastolic blood pressure & $75(71-140)$ & $70(64-77)$ & 0.131 \\
\hline Pulse rate & $87(78-104)$ & $99(88-115)$ & 0.099 \\
\hline Respiration rate & $19(18-20)$ & $20(18-20)$ & 0.700 \\
\hline Body temperature & $36.5(36.0-36.5)$ & $36.4(36.2-36.8)$ & 0.691 \\
\hline Glasgow Coma Scale & $15(13-15)$ & $12(7-13)$ & 0.049 \\
\hline $\begin{array}{l}\text { Interval from the time of } \\
\text { ingestion to ED arrival (hr) }\end{array}$ & $1.7(1.0-4.0)$ & $2.2(1.5-7.5)$ & 0.462 \\
\hline Hospital transportation method & & & 0.368 \\
\hline Paramedic ambulance & $927(71.7)$ & $11(91.7)$ & \\
\hline Hospital ambulance & $97(7.5)$ & $1(8.3)$ & \\
\hline Automobile & $267(20.7)$ & $0(0)$ & \\
\hline Ambulatory & $1(0.1)$ & $0(0)$ & \\
\hline Poisoning severity score & & & $<0.001$ \\
\hline 1 & $97(7.5)$ & $0(0)$ & \\
\hline 2 & $962(74.5)$ & $0(0)$ & \\
\hline 3 & $171(13.2)$ & $2(16.7)$ & \\
\hline 4 & $62(4.8)$ & 10 (83.3) & \\
\hline Poisoning severity score group & & & $<0.001$ \\
\hline 0 & $0(0)$ & $0(0)$ & \\
\hline Low (0-2) & $1,059(82.0)$ & $0(0)$ & \\
\hline High $(3,4)$ & $233(18.0)$ & $12(100.0)$ & \\
\hline
\end{tabular}

(continued to the next) substance compared to a median of 2.2 (IQR, 1-3) substance in the survival group ( $P=0.410$ ). Besides, the GCS, PSS, ICU admission, rate of diabetes, and endotracheal intubation status differed significantly between the two groups.

\section{LR analyses}

Univariate LR analysis identified 12 factors relevant for multivariate analysis (Table 2). In the multivariate model, age, ICU admission, and endotracheal intubation status were significant predictors of mortality $(P<0.05)$ (Table 3$)$.

\section{ANN analyses}

In the BP ANN model, age, GCS score, ICU admission, and endotracheal intubation status were the crucial factors among the 12 independent variables indicated for mortality. Thus, according to the LR as well as ANN models, age, ICU admission, and endotracheal intubation status were the common risk factors.

Table 1. Continued

\begin{tabular}{|c|c|c|c|}
\hline Variable & $\begin{array}{l}\text { Survival } \\
(n=1,292)\end{array}$ & $\begin{array}{l}\text { Death } \\
(n=12)\end{array}$ & P-value \\
\hline \multicolumn{4}{|l|}{ ICU admission } \\
\hline Yes & $366(28.3)$ & $8(66.7)$ & 0.003 \\
\hline Length of ICU stay & $0(0-2)$ & $1.5(1-13)$ & 0.162 \\
\hline Sum of risk points & $0.85(0.78-2.52)$ & $4.34(1.19-6.08)$ & 0.385 \\
\hline \multicolumn{4}{|l|}{ Diabetes } \\
\hline Yes & $137(10.6)$ & $4(33.3)$ & 0.042 \\
\hline \multicolumn{4}{|l|}{ Hypertension } \\
\hline Yes & $232(18.0)$ & $4(33.3)$ & 0.315 \\
\hline \multicolumn{4}{|l|}{ Tuberculosis } \\
\hline Yes & $15(1.2)$ & $1(8.3)$ & 0.352 \\
\hline \multicolumn{4}{|l|}{ CLD } \\
\hline Yes & $18(1.4)$ & $1(8.3)$ & 0.439 \\
\hline \multicolumn{4}{|c|}{ Hepatobiliary disease } \\
\hline Yes & $13(1.0)$ & $0(0.0)$ & 0.990 \\
\hline \multicolumn{4}{|l|}{ Cancer } \\
\hline Yes & $19(1.5)$ & $1(8.3)$ & 0.452 \\
\hline \multicolumn{4}{|c|}{ Major depression disorder } \\
\hline Yes & $224(17.3)$ & $1(8.3)$ & 0.671 \\
\hline \multicolumn{4}{|c|}{ Coronary artery disease } \\
\hline Yes & $14(1.1)$ & $0(0)$ & 1.000 \\
\hline \multicolumn{4}{|c|}{ Cerebrovascular disease } \\
\hline Yes & $7(0.5)$ & $0(0)$ & 1.000 \\
\hline \multicolumn{4}{|c|}{ Endotracheal intubation status } \\
\hline Yes & $84(6.5)$ & $7(58.3)$ & $<0.001$ \\
\hline \multicolumn{4}{|l|}{ Gastric lavage } \\
\hline Yes & $433(33.5)$ & $7(58.3)$ & 0.130 \\
\hline \multicolumn{4}{|l|}{ Charcoal } \\
\hline Yes & 647 (50.1) & $6(50.0)$ & 1.000 \\
\hline
\end{tabular}

Values are presented as median (interquartile range) or as number (\%). $E D$, emergency department; ICU, intensive care unit; CLD, chronic lung disease including asthma and chronic obstructive pulmonary disease. 
Table 2. Results of univariate logistic regression analysis for mortality after acute poisoning

\begin{tabular}{|c|c|c|c|}
\hline Variable & $\mathrm{OR}$ & $95 \% \mathrm{Cl}$ & P-value \\
\hline Age & 1.05 & $1.02-1.09$ & $<0.001$ \\
\hline Sex & 2.02 & $0.63-6.49$ & 0.221 \\
\hline No. of poisoning substances & 1.21 & $0.06-3.21$ & 0.406 \\
\hline \multicolumn{4}{|l|}{ Vital signs } \\
\hline Systolic blood pressure & 1.00 & $0.98-1.02$ & 0.812 \\
\hline Diastolic blood pressure & 0.98 & $0.95-1.01$ & 0.123 \\
\hline Pulse rate & 1.01 & $0.99-1.01$ & 0.154 \\
\hline Respiration rate & 0.95 & $0.80-1.07$ & 0.653 \\
\hline Body temperature & 0.98 & $0.83-2.15$ & 0.902 \\
\hline Glasgow Coma Scale & 0.83 & $0.74-0.95$ & $<0.001$ \\
\hline $\begin{array}{l}\text { Interval from the time of } \\
\text { ingestion to ED arrival }\end{array}$ & 0.98 & $0.85-1.05$ & 0.699 \\
\hline Hospital transport method & 0.31 & $0.02-0.96$ & 0.154 \\
\hline ICU admission & 7.74 & $0.41-43.82$ & 0.022 \\
\hline Length of ICU stay & 3.24 & $1.11-5.42$ & 0.210 \\
\hline Sum of risk points & 1.00 & $1.00-1.00$ & 0.119 \\
\hline Diabetes & 4.22 & $1.11-13.57$ & 0.047 \\
\hline Hypertension & 2.28 & $0.61-7.32$ & 0.187 \\
\hline Tuberculosis & 1.25 & $0.32-4.32$ & 0.256 \\
\hline CLD & 8.94 & $1.07-74.42$ & 1.000 \\
\hline Cancer & 6.09 & $0.33-33.79$ & 0.092 \\
\hline Major depressive disorder & 0.43 & $0.02-2.24$ & 0.422 \\
\hline Coronary artery disease & 0.00 & $0.00-0.00$ & 1.000 \\
\hline Cerebrovascular disease & 0.00 & $0.00-0.00$ & 1.000 \\
\hline Endotracheal intubation & 20.13 & $6.3-69.3$ & $<0.001$ \\
\hline Gastric lavage & 2.77 & $0.88-9.42$ & 0.082 \\
\hline Charcoal & 1.00 & $0.31-3.20$ & 0.901 \\
\hline
\end{tabular}

$\mathrm{OR}$, odds ratio; $\mathrm{Cl}$, confidence interval; $\mathrm{ED}$, emergency department; ICU, intensive care unit; CLD, chronic lung disease including asthma and chronic obstructive pulmonary disease.

Table 3. Results of multivariate logistic regression for mortality after acute poisoning

\begin{tabular}{lcccr}
\hline Variable & Estimate & Adjusted OR & $95 \% \mathrm{Cl}$ & P-value \\
\hline Age & 0.45 & 5.81 & $3.21-9.01$ & 0.032 \\
Endotracheal intubation & 2.35 & 20.13 & $6.25-64.79$ & $<0.001$ \\
ICU admission & 0.35 & 5.06 & $1.51-16.91$ & 0.031
\end{tabular}

$\mathrm{OR}$, odds ratio; $\mathrm{Cl}$, confidence interval; ICU, intensive care unit.

\section{Comparison between the two models}

The area under the ROC curve (AUROC) was obtained applying the two models constructed using the test data set for mortality identification (Fig. 2). The accuracy of mortality prediction was evaluated through ROC curve analysis, comparing those of the BP ANN and LR models. The AUROC of the BP ANN model was 0.88 $(95 \% \mathrm{Cl}, 0.77-1.00)$, which was significantly higher than that of the LR model $(0.74 ; 95 \% \mathrm{Cl}, 0.59-0.90)(\mathrm{P}=0.042)$ (Table 4).
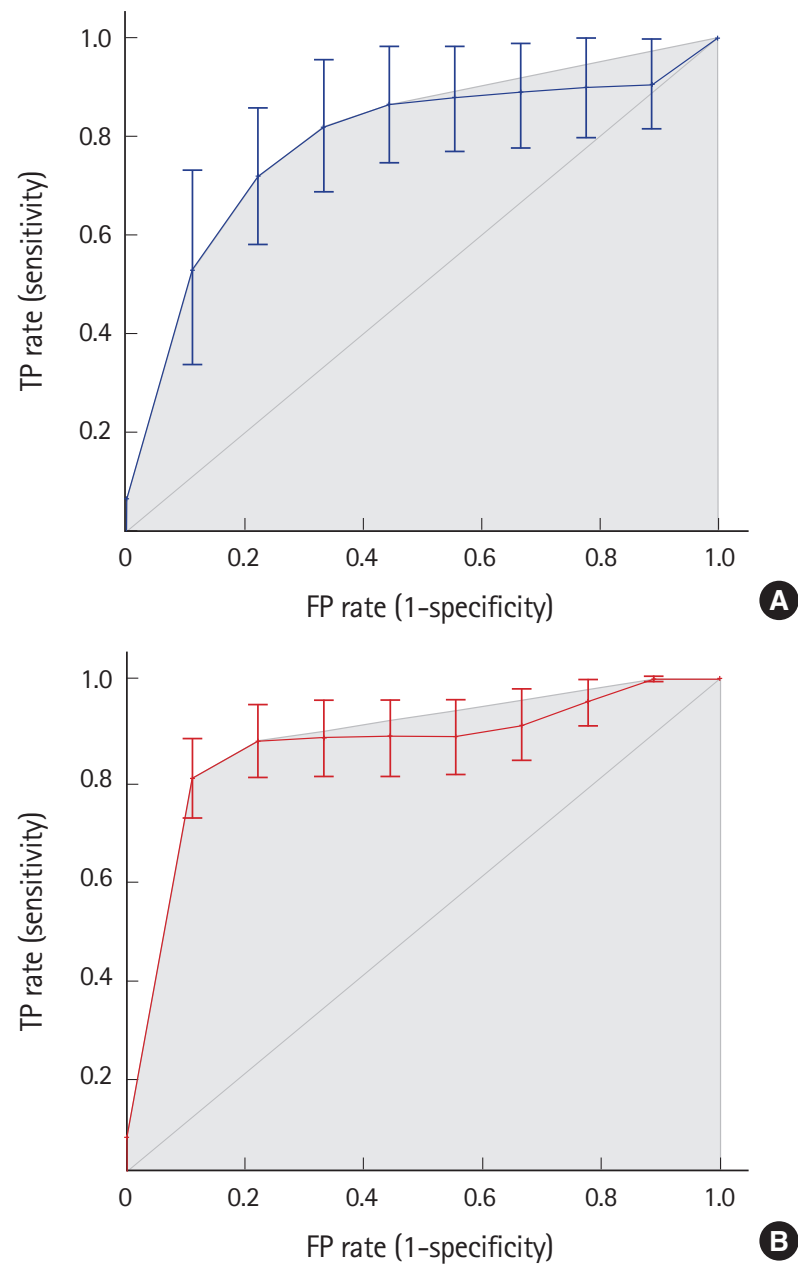

Fig. 2. Receiver operating characteristics curves for in-hospital mortality after acute poisoning constructed using (A) logistic regression model and $(B)$ artificial neural network model. The area under receiver operating characteristics curves were $0.74,0.88$ for logistic regression and artificial neural network models. TP, true positive; FP, false positive.

Table 4. Performance comparison of LR and ANN models for predicting in-hospital mortality after acute poisoning

\begin{tabular}{lcccc}
\hline & AUC & $95 \% \mathrm{Cl}$ & CA & Precision \\
\hline LR & 0.74 & $0.59-0.90$ & 0.99 & 0.98 \\
ANN & 0.88 & $0.77-1.00$ & 0.99 & 0.98
\end{tabular}

$L R$, logistic regression; $A N N$, artificial neural network; $A U C$, area under curve; $C l$ confidence interval; $C A$, classification accuracy.

\section{DISCUSSION}

The ability to predict the exact risk of mortality at an early stage during the treatment of critical patients in the ED is crucial for patient triage and improving outcomes. It has been demonstrated that the ANN model has good prognosis prediction for numerous disease conditions and superiority over conventional predictive 
models, even when the same input variables are used for model generation. ${ }^{7}$ The findings of this study confirm that the ANN model predicts the mortality of AP patients in the ED more accurately than $L R$.

A preceding study on critical patients with sepsis treated in the ED indicated that the ANN could predict the possibility of death more precisely than LR. ${ }^{13}$ Another study on patients presented in the ED with acute coronary syndrome also showed better prognosis prediction with an ANN ensemble model, compared to an LR model. ${ }^{14}$ In a study comparing the ability of an ANN model with that of a severity scoring system, the acute physiology and chronic health evaluation II score (APACHE II), for predicting the mortality of patients admitted to the general ICU, the ANN model predicted 336 among the 337 deaths, whereas the APACHE II predicted 246 deaths. ${ }^{15}$

The ANN algorithm possesses an automatic handling function for missing values and performs feature selection enabling the model to perform comparable functions as the LR model, requiring considerably less effort. Another advantage of the ANN model is that preprocessing is not necessary. The LR model has a relatively simple approach for modeling NNs employing a fully connected layer of the feed-forward network without hidden layers, using a sigmoid activation function. Thus, the ANN can potentially model more complicated nonlinear relationships than the LR. ${ }^{16}$ However, a limitation of ANN modeling is the requirement for state-of-the-art software, in addition to the considerable computer resources for training and testing NNs.

In comparison, the LR model can clarify the variables that provide the strongest predictability of an outcome based on the magnitude of the coefficients and the associated ORs. Moreover, LR analysis can eliminate independent variables that are not related to a particular outcome of interest through a stepwise variable selection process, whereas the ANN model may contain several unimportant predictor variables that may remain unrecognized, compromising or complicating model application. The existence of a statistical relationship between a predictor variable and an outcome alone in an ANN model does not imply causality. LR models are superior to NNs in identifying possible causal relationships. ${ }^{8}$ This lack of interpretability at an individual predictor level is one of the most criticized features of ANN models. ${ }^{16}$ Furthermore, ANNs exhibit difficulties with generalization, producing models that can overfit data. To improve generalization, internal cross validation was performed in this study; however, external validation may also have been beneficial.

Previous studies have shown that among patients with $A P$, older individuals were more prone to death or prolonged ICU stay, with each 10-year increase in age associated with a 0.36 increase in the OR for death. ${ }^{17,18}$ The results of this study also indicate that age is a critical factor for death following AP, with eight of the twelve deaths occurring in patients aged 60 or older. Age was identified as a common risk factor for mortality by the LR as well as ANN models.

Five PSS grades ( 0 , none; 4 , fatal poisoning) were created for symptoms or signs of organ failure following AP. ${ }^{10}$ PSS generates a very high AUROC value for death risk because all the causes of death are grade 4 on this scale. PSS needs to consider the overall clinical progression and must be applied according to the most severe symptomatology, and the clinician needs to collect considerable data from the 12 organ systems. As PSS scores are subjective and have low interperson reliability because judgments such as mild, moderate, and prolonged can be confounded, ${ }_{1}^{19}$ it was excluded from the independent variables when comparing death predictability. PSS was used only as a dependent variable to score risk points for toxic substances in order to measure the risk score in patients with suspected ingestion of multiple substances in AP.

The number of toxic substances did not constitute a significant factor in mortality prediction, even though toxic substances received four points from the PSS as the associated complications are highly related to death. Patients with AP due to multiple agents or mixed drug overdoses are common in the ED. A study from Taiwan showed that 208/1,507 (13.8\%) patients were exposed to more than one agent. ${ }^{20}$ In addition, the study showed that patients ingested a median of 1 (range, 1-16) type of substance and that 569/1,304 (43.6\%) patients consumed two or more medications on average..$^{20}$ Patients with mixed poisoning constitute a considerable challenge to ED staff and present increased difficulty with regard to decontamination and treatment compared to poisoning with a single agent. Rogers and Heard ${ }^{18}$ found an increase in the relative rate of death ranging from 1.2 to 7.1 for each poisoning agent with each 10-year increase in age. In this study, neither the number of poisoning substances nor the SRP of each ingested substance was significantly associated with mortality. Further research is required to clarify the reasons for this discrepancy.

This study has several limitations, including small sample size and relatively sporadic outcome events. However, more than 10 outcome events for each independent variable are generally acceptable. ${ }^{21}$ The samples used in our model are appropriate because 12 events were observed in 1,304 individuals for a single outcome variable. ${ }^{22}$ Clermont et al. ${ }^{23}$ designed a prospective study with a small sample size of 1,647 patients in intensive care. They compared the LR and ANN models using a random training set of 1,200 patients and the remaining 447 patients as the validation set. They iterated model construction on progressively smaller 
training sets and repeated prediction with the same validation set. As the size of the training set decreased, the performance of the model with the validation set decreased rapidly, demonstrating poor fit with under 800 samples. In comparison, this study utilized 1,292 samples, with 912 patients used as the training set to ensure proper fit.

Liisanantti et al..$^{24}$ reported that respiratory and renal dysfunction or failure following AP are among the risk factors for in-hospital mortality. However, the parameters from the laboratory data for renal, cardiovascular, and respiratory dysfunction were not included in this study. The focus of this study was not to identify the risk factors in order to compare LR and ANN for death prediction. As mentioned previously, such lack of interpretability at the level of individual predictors is one of the most criticized features of ANN models. ${ }^{16}$

A predictive tool is urgently required to estimate the mortality risk for patients with $A P$ in the $E D$, which can serve as a guideline for medical decisions and patient disposition. In this study, a novel ANN model was developed and validated to determine the mortality risk for patients with AP during initial ED assessment. Trained ANNs approach the functionality of a cluster in fundamental manner, which we expect would perform triage in EDs as effectively as LR and provide an additional tool for efficient resource and human resource management. Nevertheless, further optimization of the model is necessary for more accurate predictions.

\section{CONFLICT OF INTEREST}

No potential conflict of interest relevant to this article was reported.

\section{ACKNOWLEDGMENTS}

This work was supported by The Catholic University of Korea Daejeon St. Mary's Hospital. The Clinical Research Institute Grant was funded by The Catholic University of Korea Daejeon St. Mary's Hospital (CMCDJ-P-2021-013).

\section{REFERENCES}

1. Warner $M$, Chen LH, Makuc DM, Anderson RN, Minino AM. Drug poisoning deaths in the United States, 1980-2008. NCHS Data Brief 2011:1-8.

2. Tang $Y$, Zhang L, Pan J, et al. Unintentional poisoning in China, 1990 to 2015: the global burden of disease study 2015. Am J Public Health 2017;107:1311-5.

3. Strom J, Thisted B, Krantz T, Bredgaard Sorensen M. Self-poi- soning treated in an ICU: drug pattern, acute mortality and short-term survival. Acta Anaesthesiol Scand 1986;30:148-53.

4. Maignan $\mathrm{M}$, Pommier $\mathrm{P}$, Clot $\mathrm{S}$, et al. Deliberate drug poisoning with slight symptoms on admission: are there predictive factors for intensive care unit referral? A three-year retrospective study. Basic Clin Pharmacol Toxicol 2014;114:281-7.

5. Corcoran C, Mehta C, Patel N, Senchaudhuri P. Computational tools for exact conditional logistic regression. Stat Med 2001; 20:2723-39.

6. King G, Zeng L. Logistic regression in rare events data. Polit Anal 2001;9:137-63.

7. Patel JL, Goyal RK. Applications of artificial neural networks in medical science. Curr Clin Pharmacol 2007;2:217-26.

8. Ripley BD. Neural networks and related methods for classification. J R Stat Soc Series B Stat Methodol 1994;56:409-37.

9. Sargent DJ. Comparison of artificial neural networks with other statistical approaches: results from medical data sets. Cancer 2001;91:1636-42.

10. Persson HE, Sjoberg GK, Haines JA, Pronczuk de Garbino J. Poisoning severity score: grading of acute poisoning. J Toxicol Clin Toxicol 1998;36:205-13.

11. World Health Organization. International statistical classification of diseases and related health problems. Geneva: World Health Organization; 2004.

12. Hahnloser RH, Sarpeshkar R, Mahowald MA, Douglas RJ, Seung HS. Digital selection and analogue amplification coexist in a cortex-inspired silicon circuit. Nature 2000;405:947-51.

13. Jaimes F, Farbiarz J, Alvarez D, Martinez C. Comparison between logistic regression and neural networks to predict death in patients with suspected sepsis in the emergency room. Crit Care 2005;9:R150-6.

14. Green M, Bjork J, Forberg J, Ekelund U, Edenbrandt L, Ohlsson M. Comparison between neural networks and multiple logistic regression to predict acute coronary syndrome in the emergency room. Artif Intell Med 2006;38:305-18.

15. Nimgaonkar A, Karnad DR, Sudarshan S, Ohno-Machado L, Kohane I. Prediction of mortality in an Indian intensive care unit. Comparison between APACHE II and artificial neural networks. Intensive Care Med 2004;30:248-53.

16. Tu JV. Advantages and disadvantages of using artificial neural networks versus logistic regression for predicting medical outcomes. J Clin Epidemiol 1996;49:1225-31.

17. Turnheim K. When drug therapy gets old: pharmacokinetics and pharmacodynamics in the elderly. Exp Gerontol 2003;38: 843-53.

18. Rogers JJ, Heard K. Does age matter? Comparing case fatality rates for selected poisonings reported to U.S. poison centers. 
Clin Toxicol (Phila) 2007;45:705-8.

19. Schwarz ES, Kopec KT, Wiegand TJ, Wax PM, Brent J. Should we be using the poisoning severity score? J Med Toxicol 2017; 13:135-45.

20. Lee $\mathrm{HL}$, Lin $\mathrm{HJ}$, Yeh SY, Chi CH, Guo HR. Etiology and outcome of patients presenting for poisoning to the emergency department in Taiwan: a prospective study. Hum Exp Toxicol 2008; 27:373-9.

21. Concato J, Peduzzi P, Holford TR, Feinstein AR. Importance of events per independent variable in proportional hazards analysis. I. Background, goals, and general strategy. J Clin Epidemiol 1995;48:1495-501.
22. Concato J, Feinstein AR, Holford TR. The risk of determining risk with multivariable models. Ann Intern Med 1993;118: 201-10.

23. Clermont G, Angus DC, DiRusso SM, Griffin M, Linde-Zwirble WT. Predicting hospital mortality for patients in the intensive care unit: a comparison of artificial neural networks with logistic regression models. Crit Care Med 2001;29:291-6.

24. Liisanantti JH, Ohtonen P, Kiviniemi O, Laurila JJ, Ala-Kokko TI. Risk factors for prolonged intensive care unit stay and hospital mortality in acute drug-poisoned patients: an evaluation of the physiologic and laboratory parameters on admission. J Crit Care $2011 ; 26: 160-5$. 